\title{
Advancing texture metrics to model landscape heterogeneity
}

Schuh, Leila ; Furrer, Reinhard ; Schaepman, Michael E ; Santos, Maria J ; de Jong, Rogier

\begin{abstract}
We advance methods to study landscape heterogeneity and assess the suitability of these methods to detect boundary regions between land cover types. We employ 2nd-order texture metrics on continuous and on discrete vegetation data in North Eurasia and on a smaller region therein. Furthermore, we advance 2nd-order texture metrics to incorporate spatial scales in novel ways. The metrics entropy, contrast, and homogeneity are found to detect boundary regions well on discrete data in the smaller study area, but create fuzzy results on continuous data and when models are run for the whole of northern Eurasia. The scales we consider affect the size of the boundary regions, but also the metric values. The results elucidate the need for aligned scale determination and data restriction procedures. Our approach offers new opportunities to model landscape heterogeneity across scales.
\end{abstract}

DOI: https://doi.org/10.1109/igarss39084.2020.9323930

Posted at the Zurich Open Repository and Archive, University of Zurich

ZORA URL: https://doi.org/10.5167/uzh-202285

Conference or Workshop Item

Published Version

Originally published at:

Schuh, Leila; Furrer, Reinhard; Schaepman, Michael E; Santos, Maria J; de Jong, Rogier (2020). Advancing texture metrics to model landscape heterogeneity. In: IGARSS 2020 - 2020 IEEE International Geoscience and Remote Sensing Symposium, Waikoloa, HI, USA, 26 September 2020 - 2 October 2020, 2735-2738.

DOI: https://doi.org/10.1109/igarss39084.2020.9323930 


\title{
ADVANCING TEXTURE METRICS TO MODEL LANDSCAPE HETEROGENEITY
}

\author{
Leila Schuh ${ }^{1}$, Michael Schaepman ${ }^{2}$, Maria J. Santos ${ }^{3}$, Rogier de Jong ${ }^{4}$, Reinhard Furrer ${ }^{1}$ \\ ${ }^{1}$ Department of Mathematics, Applied Statistics, University of Zurich, Switzerland \\ ${ }^{2}$ Department of Geography, Remote Sensing Laboratories, University of Zurich, Switzerland \\ ${ }^{3}$ Department of Geography, Earth System Sciences, University of Zurich, Switzerland \\ ${ }^{4}$ Swiss Re Institute, Swiss Re Management Ltd, Zurich, Switzerland
}

\begin{abstract}
We advance methods to study landscape heterogeneity and assess the suitability of these methods to detect boundary regions between land cover types. We employ $2^{\text {nd }}$-order texture metrics on continuous and on discrete vegetation data in North Eurasia and on a smaller region therein. Furthermore, we advance $2^{\text {nd }}$-order texture metrics to incorporate spatial scales in novel ways. The metrics entropy, contrast, and homogeneity are found to detect boundary regions well on discrete data in the smaller study area, but create fuzzy results on continuous data and when models are run for the whole of northern Eurasia. The scales we consider affect the size of the boundary regions, but also the metric values. The results elucidate the need for aligned scale determination and data restriction procedures. Our approach offers new opportunities to model landscape heterogeneity across scales.
\end{abstract}

Index Terms - Between type heterogeneity, within type heterogeneity, $2^{\text {nd }}$-order texture metrics, entropy, contrast, homogeneity, boundary regions, scale.

\section{INTRODUCTION}

Spatial structure is a driving force to the earth's environmental, ecological, and social processes that occur on local, regional and global scales $[1,5,6]$. We explore landscape heterogeneity, which has been quantified with different measures and is, thus, associated with more than one meaning $[13,14]$. Essentially, the concept is independent of the research context, and implies a spatially explicit, horizontal dimension. The study of landscape heterogeneity often involves classification of land cover types [3,14,17]. In this context, landscape heterogeneity can be conceptualized as between type heterogeneity. Conversely, vegetation structure and plant diversity are measures of within type heterogeneity $[14,15]$. An exciting challenge arises from the fact that, especially from remote sensing sources, we are provided with large amounts of continuous data like the Normalized Difference Vegetation Index
(NDVI), which contains information about both, betweenand within type heterogeneity.

Biomes such as Tundra and Taiga in the northern high latitudes are expected to follow their potential ranges, which shift in space due to changing environmental conditions $[4,5]$. The boundary regions between biomes are, thus, areas of interest for the study of vegetation response to climate change. While texture metrics have found much of their application as classification methods [3,10,11,17], we employ them to identify boundary regions between classes. Furthermore, questions of scale complicate the study of landscape heterogeneity. Any spatial structure occurs on a specific scale $[1,2,4,9,13,16,17]$. Typically, we find several dominant scales in remote sensing imagery and the optimal scale corresponds to the research question. We take a datadriven perspective and claim that if we are able quantify heterogeneity on different scales, we can inform the study of environmental, ecological, and social processes.

We use geographical raster imagery and methods that have been designed to process gridded fields. Originally developed for image processing, texture metrics have been used to study heterogeneity in remote sensing data $[7,10,11,15,17]$. So-called $1^{\text {st }}$-order metrics are descriptive statistics of frequency distributions within a pixel neighborhood [15,17]. Examples include evenness, mean, variance, and the coefficient of variation. These measures do not account for pixel positions within the image. $2^{\text {nd }}$-order texture metrics consider how often pixel values occur together, at specified angles and distances [7,9,10,15,17]. Thus, they provide measures of spatial heterogeneity that account for the relative position of pixel values to each other.

We assume the boundary regions between types be characterized by high levels of heterogeneity, whereas low heterogeneity levels are more likely to represent types. We further assume that the results will differ depending on the scales we consider. We employ $2^{\text {nd }}$-order texture metrics to identify boundary regions between land cover types using continuous and discretized data. We further advance $2^{\text {nd }}$ order texture metrics to incorporate scale in novel ways. 


\section{METHODS}

A whole suite of texture metrics exists, and in some cases the same metric name has been used for slightly different equations. Contrast, dissimilarity, and homogeneity are socalled contrast measures. Contrast and dissimilarity both highlight pairs with spectrally different pixel values. If both values are the same, contrast and dissimilarity will be 0 . Weights increase with the difference between values, exponentially for contrast and linearly for dissimilarity $[7,9,10,15,17]$. The homogeneity measure accentuates the opposite and becomes 1 when both pixel values are identical. We employ contrast and homogeneity.

Angular second moment, maximum probability, and entropy have been used as measures of disorderliness [17]. While all $2^{\text {nd }}$-oder measures account for pixel positions by considering pairs, orderliness measures give an indication of how regularly the pairs are placed in space $[9,15,17]$. We use entropy, which has been found one of the most powerful metrics in different research contexts $[3,11,15,17]$. High entropy values indicate low levels of orderliness.

When we talk about considering scale, we must distinguish scale through a context and methods perspective. We define scale from the perspective of $2^{\text {nd }}$-order heterogeneity metrics as the number of pixels considered, the pixel neighborhood [9]. The whole image represents the largest scale we can take into account. To define spatial scale in an environmental, ecological, or social context, we would need to include pixel resolution and geographic location.

$2^{\text {nd }}$-order metrics are based on a pixel co-occurrence matrix, which contains information about the frequency of pixel values occurring as pairs at certain angles and distances. The matrix is defined for four different angles: horizontal $\left(0^{\circ}\right)$, $45^{\circ}$, vertical $\left(90^{\circ}\right)$ and $135^{\circ}$. The distance can be any integer, with distance 1 referring to adjacent pixels, distance 2 referring to neighbors with one pixel in-between, and so forth $[7,9,10,17]$.

Let $n$ be the number of unique pixel values that occur in the image. The dimensions of the corresponding co-occurrence matrix are $n$ rows and $n$ columns. The matrix is normalized by the number of possible pixel pairs, which can differ with direction. Therefore, the co-occurrence matrix is a frequency table that contains the empirical probabilities of pixel pair occurrences at specified angles and distances within a certain neighborhood $[7,9,17]$.

The co-occurrence matrix is passed to the metric equations. Thus, heterogeneity is calculated in relation to the empirical probabilities of pixel pair co-occurrences, observed on the respective scale. Traditionally, the metrics were calculated for the whole image, a procedure that becomes computationally expensive with large numbers of pixels and unique pixel values $[7,9,10,17]$. Tunamu and Jetz model heterogeneity on a global level by calculating the metric for smaller neighborhoods and assigning the final heterogeneity value to larger grid cells [15]. We apply texture metrics with a moving window approach, assigning the final heterogeneity value to the center pixel [9]. This allows us to retain the original pixel resolution.

Depending on the research context, neither the empirical probabilities of pixel associations in the whole image, nor in a local neighborhood, may be the most informative $[2,16]$. We use an adaptable combination scheme of scales, which considers different levels of detail, ranging from local neighborhood optimization for individual pixels to the largest structures in the image. Furthermore, we disentangle the neighborhoods associated with the co-occurrence matrix and with the heterogeneity measure. We can then calculate heterogeneity for a certain neighborhood, given the empirical probability of pixel associations on a larger scale. With our approach, different scales can be considered at the same time.

We test different neighborhood combinations on two remote sensing images. One image represents North Eurasia stretching from $50^{\circ}$ to $70^{\circ}$ North, and $-25^{\circ}$ West to $180^{\circ}$ East, with dimensions 1100 rows, 22800 columns, and 25080000 cells (Fig.4). A smaller image represents a study region within North Eurasia with 221 rows, 1092 columns, and 241332 cells (Figs.1\&4). To consider medium scales within North Eurasia, we divide the image into sections and treat these as whole images. We calculate the co-occurrence matrix for each of the sections, and heterogeneity for a smaller scale.

We use Modis NDVI yearly averages over the growing season as continuous data, and a classification product thereof, based on characteristic NDVI thresholds for Tundra and Taiga biomes (Fig.1) [4]. However, we do not claim an ideal classification, but rather produce discrete data that is based only on the original data. This allows us to evaluate the ability of the methods to detect boundary regions between types, which can easily be identified in discrete data. We calculate heterogeneity using the metrics entropy, contrast and homogeneity, similar to Haralick et. al and Tuanmu and Jetz $[10,15]$. We assess the suitability of these metrics to detect boundary regions on both data types and images, with different neighborhood combinations to calculate the empirical probability of pixel co-occurrences and the heterogeneity metric. We consider directly adjacent pixel pairs in horizontal direction. For data pre-processing and download we use google earth engine [8]. All data analysis in done in R [12].

\section{RESULTS}

Calculations for the smaller study region reveal different results, depending on the input data. The heterogeneity maps calculated on discrete data display clear boundary regions (Fig.2\&3). The clearest images are generated when the metric entropy is applied (Fig.2). Continuous data leads to fuzzy results where boundary regions cannot be detected (Fig.3). Using categorical data on the whole of North Eurasia, the results show structure, but we cannot detect boundary regions (Fig.4). The level of detail in the heterogeneity maps is affected by the choice of both scales. 
Figure 2 exemplifies that the neighborhoods considered for the calculation of the co-occurrence matrix, and for the heterogeneity metric, not only influence the size of boundary areas, but also the values of the metric itself. In the heterogeneity map of northern Eurasia, we see strong edge effects, which result from the choice of co-occurrence neighborhoods (Fig.4).

\section{CONCLUSIONS}

We conclude that out of the three metrics, entropy is the most suitable to detect boundary regions. When categorical data is used, boundary areas can be identified by high levels of heterogeneity and land cover types are characterized by low levels of heterogeneity. However, the ability to detect boundary regions decreases with increasing numbers of pixels, but also with increasing numbers of unique values; which leaves room for further methodological advancement. Furthermore, the scales we employ, and the way we consider them, influences the results. On the smaller study area, we can conclude that restricting the number of unique values in the original image enables the detection of boundary regions. However, this was not a sufficient solution when modeling over the whole of North Eurasia. The neighborhoods we use to model landscape heterogeneity must fit the image and the context, in our case the detection of boundary regions.

While no automated method exists to model landscape heterogeneity with context optimized scales and data, we could show that calculating texture metrics for a smaller neighborhood, in relation to pixel co-occurrences on a larger area, is a promising approach to model landscape heterogeneity across scales.

a)

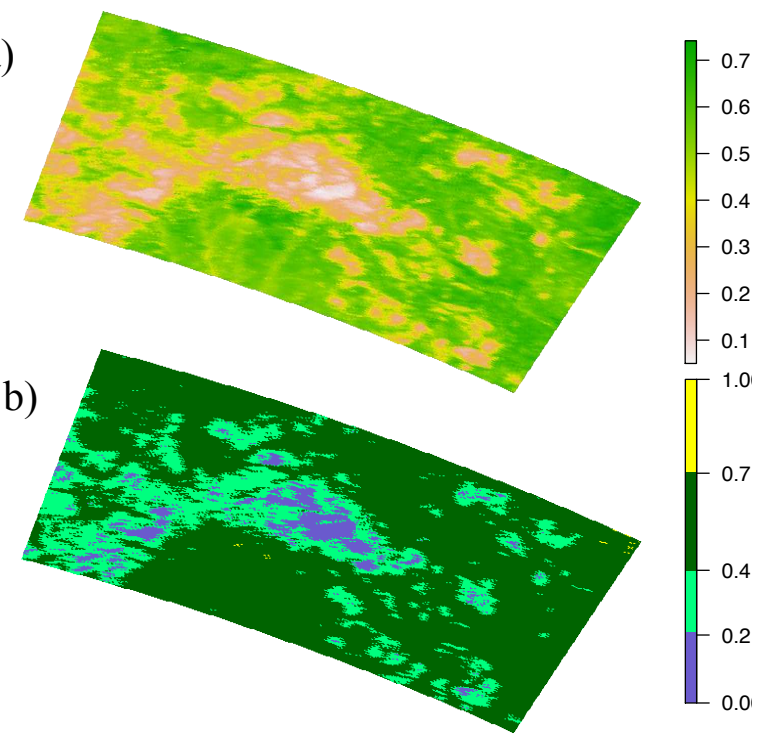

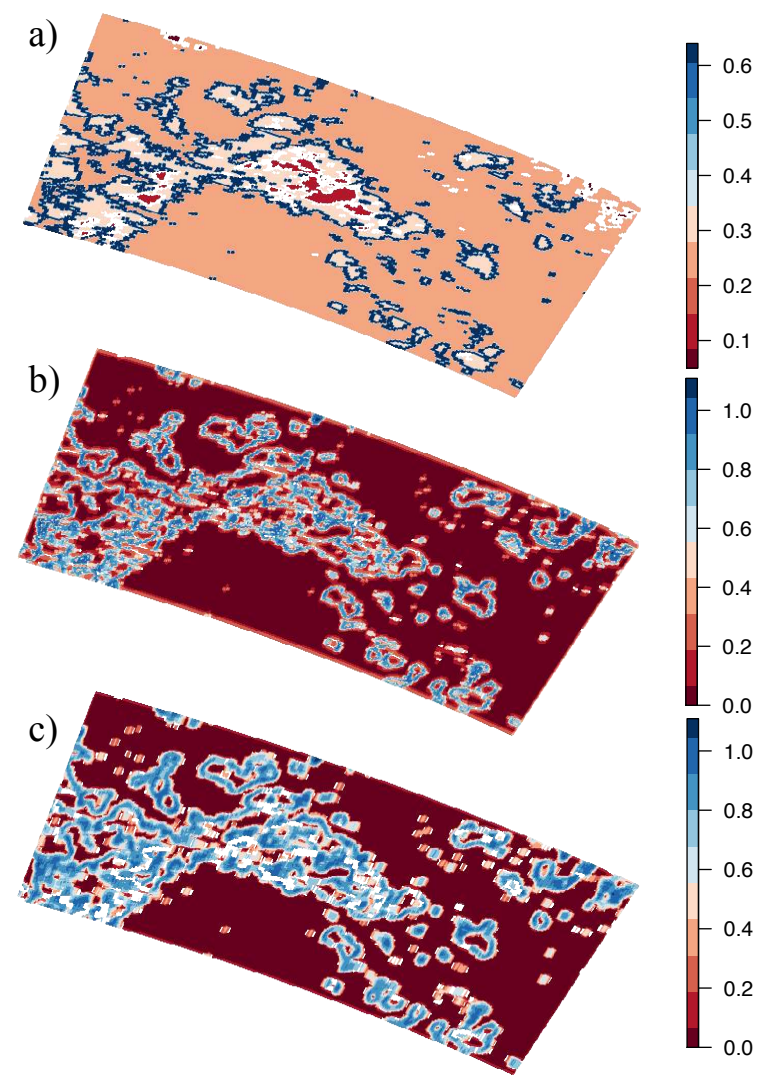

Figure 2. Entropy (discrete NDVI) for different combinations of empirical probability neighborhood (NgbProb) and heterogeneity neighborhood (NgbHet). a) NgbProb whole image \& NgbHet 3x3. b) NgbProb 11x11 \& NgbHet 3x3. c) NgbProb 11x11 \& NgbHet 7x7.

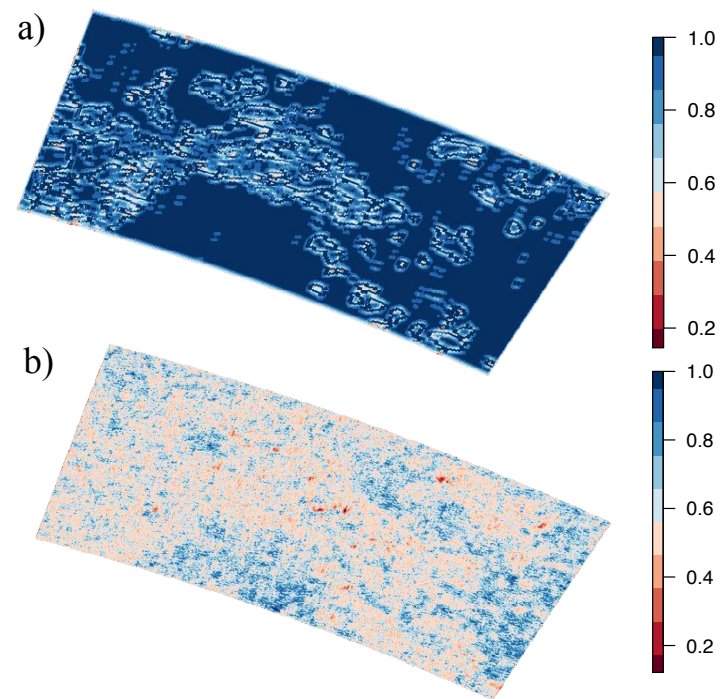

Figure 3. Homogeneity. a) discrete NDVI. NgbProb 11x11 \& NgbHet 3x3. b) continuous NDVI. NgbProb 5x5 \& NgbHet $3 \times 3$.

Figure 1. a) continuous NDVI. b) discrete NDVI. Classification based on Defries \& Townshed, 1994. Blue: NDVI $<0.2$, light green: $0.2 \leq \mathrm{NDVI} \leq$ 0.4 , dark green: $0.4<\mathrm{NDVI} \leq 0.7$, yellow: $0.7<\mathrm{NDVI} \leq 1$. 


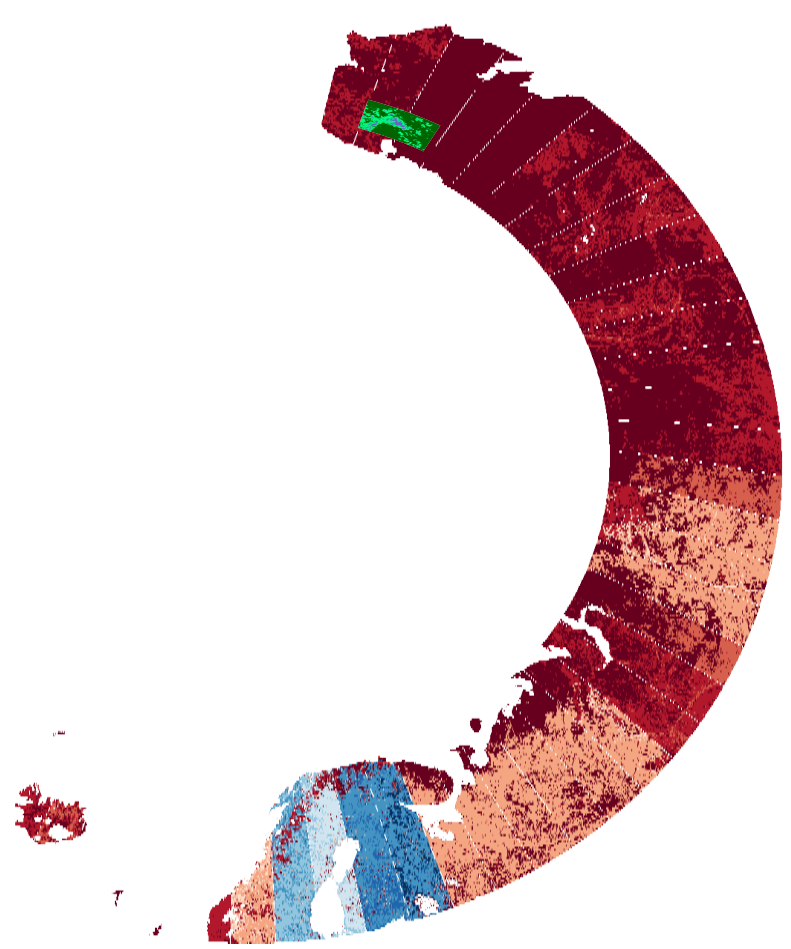

Figure 4: Contrast (discrete NDVI), North Eurasia. NgbProb 1100x600, NgbHet $3 \times 3$. The smaller study area is visible in the North-East.

\section{ACKNOWLEDGEMENTS}

This study is kindly supported by the University of Zurich Research Priority Program Global Change and Biodiversity.

\section{REFERENCES}

[1] Amelina, A., "Socio-spatial scales as social boundaries? Or: How do migration studies profit from including 'space' in the sociology of social boundaries", Migration Letters, 3, pp. 273- 288.

[2] Cale, P., and Hobbs, R.J., "Landscape heterogeneity indices: problems of scale and applicability, with particular reference to animal habitat description", Pacific Conservation Biology, 1(December), pp. 183-193, 1994.

[3] Clausi, D.A., "Comparison and fusion of co-occurrence, Gabor and MRF texture features for classification of SAR sea-ice imagery", Atmosphere-Ocean, 39 (3), pp. 183-194, 2001.

[4] Defries, R.S., and Townshed, J.R.G., "NDVI-derived land cover classifications at a global scale", International Journal of Remote Sensing, 15(17), pp. 3567-3586, 1994.

[5] De Jong, R., Verbesselt, J., Zeileis, A., and Schaepman, M.E., "Shifts in global vegetation activity trends." Remote Sensing, 5(3), pp. 1117-1133, 2013.

[6] De Jong, R., Schaepman, M.E. , Furrer, R., De Bruin, S., and Verburg, P. H., "Spatial relationship between climatologies and changes in global vegetation activity", Global Change Biology, 19(6), pp. 1953-1964, 2013.

[7] Gonzales, R.C., and Woods, R.E., Digital Image Processing. $3^{\text {rd }}$ edition, Prentice Hall, Upper Saddle River, N.J, 2008.

[8] Gorelick, N., Hancher, M., Dixon, M., Ilyushchenko, S., Thau, D., \& Moore, R., "Google Earth Engine: Planetaryscale geospatial analysis for everyone", Remote Sensing of Environment, 202, pp. 18-27, 2017.

[9] Hall-Beyer, M, "Practical guidelines for choosing GLCM textures to use in landscape classification tasks over a range of moderate spatial scales", International Journal of Remote Sensing, 38(5) pp. 1312-1338, 2017.

[10] Haralick, R.M. , Shanmugam, K., and Dinstein, I.H., "Textural features for image classification", IEEE Transactions on Systems, Man and Cybernetics, 3, pp. 610 621, 1973.

[11] Puissant, A., Hirsch, J., and Weber, Ch., "The utility of texture analysis to improve per-pixel classification for very high spatial resolution imagery", International Journal of Remote Sensing, 26(4), pp. 733-745, 2006.

[12] R Core Team, R: A language and environment for statistical computing, $\mathrm{R}$ Foundation for Statistical Computing, Vienna, Austria, https://www.R-project.org, 2017.

[13] Stein, A., Gerstner, K., and Kreft, H., "Environmental heterogeneity as a universal driver of species richness across taxa, biomes and spatial scales", Ecology Letters, 17(7), pp. 866-880, 2014.

[14] Stein, A., and Kreft, H., "Terminology and quantification of environmental heterogeneity in speciesrichness research", Biological Reviews, 90(3), pp. 815-836, 2015.

[15] Tuanmu, M.N., and Jetz, W., "A global, remote sensing-based characterization of terrestrial habitat heterogeneity for biodiversity and ecosystem modeling", Global Ecology and Biogeography, 24(11), pp. 1329-1339, 2015.

[16] Turner, M.G., O’Neill, R.V., Gardner, R.H., and Milne, B.T., "Effects of changing spatial scale on the analysis of landscape pattern", Landscape Ecology, 3(3-4), pp. 153$162,1989$.

[17] Warner, T., "Kernel-based texture in remote sensing image classification", Geography Compass, 5(10), pp. 781798, 2011. 